\title{
Inter- and intraobserver reliability of non-weight-bearing foot radiographs compared with CT in Lisfranc injuries
}

\author{
Ville T. Ponkilainen ${ }^{1}\left(\mathbb{D} \cdot\right.$ Nikke Partio $^{2}$ - Essi E. Salonen ${ }^{2} \cdot$ Antti Riuttanen $^{2} \cdot$ Emma- Liisa Luoma $^{2}$ - Gilber Kask ${ }^{2}$.

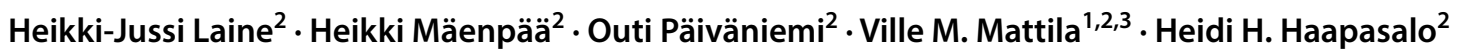

Received: 25 October 2019 / Published online: 5 March 2020

(c) The Author(s) 2020

\begin{abstract}
Background Injury of the tarsometatarsal (TMT) joint complex, known as Lisfranc injury, covers a wide range of injuries from subtle ligamentous injuries to severely displaced crush injuries. Although it is known that these injuries are commonly missed, the literature on the accuracy of the diagnostics is limited. The diagnostic accuracy of non-weight-bearing radiography (inter- or intraobserver reliability), however, has not previously been assessed among patients with Lisfranc injury.

Methods One hundred sets of foot radiographs acquired due to acute foot injury were collected and anonymised. The diagnosis of these patients was confirmed with a CT scan. In one-third of the radiographs, there was no Lisfranc injury; in onethird, a nondisplaced $(<2 \mathrm{~mm})$ injury; and in one-third, a displaced injury. The radiographs were assessed independently by three senior orthopaedic surgeons and three orthopaedic surgery residents.

Results Fleiss kappa ( $\kappa$ ) coefficient for interobserver reliability resulted in moderate correlation $\kappa=0.50$ (95\% CI: $0.45-0.55$ ) (first evaluation) and $\kappa=0.58$ (95\% CI: 0.52-0.63) (second evaluation). After three months, the evaluation was repeated and the Cohen's kappa ( $\kappa)$ coefficient for intraobserver reliability showed substantial correlation $\kappa=0.71$ (from 0.64 to 0.85 ). The mean (range) sensitivity was $76.1 \%(60.6-92.4)$ and specificity was $85.3 \%$ (52.9-100). The sensitivity of subtle injuries was lower than severe injuries ( $65.4 \%$ vs $87.1 \% p=0.003)$.

Conclusions Diagnosis of Lisfranc injury based on non-weight-bearing radiographs has moderate agreement between observers and substantial agreement between the same observer in different moments. A substantial number (24\%) of injuries are missed if only non-weight-bearing radiographs are used. Nondisplaced injuries were more commonly missed than displaced injuries, and therefore, special caution should be used when the clinical signs are subtle.
\end{abstract}

Level of evidence III.

Keywords Lisfranc $\cdot$ Injury $\cdot$ Radiographs $\cdot$ X-ray $\cdot$ Interobserver $\cdot$ Intraobserver $\cdot$ Reliability $\cdot$ Responsiveness

Ville T. Ponkilainen

ville.ponkilainen@tuni.fi

1 School of Medicine, University of Tampere, 33520 Tampere, Finland

2 Department of Orthopaedics and Traumatology, Faculty of Medicine and Life Sciences and Tampere University Hospital, University of Tampere, Teiskontie 35, PL2000, 33521 Tampere, Finland

3 COXA Hospital for Joint Replacement, Biokatu 6, 33520 Tampere, Finland

\section{Introduction}

Lisfranc injury was originally described as a partial or complete dislocation of the tarsometatarsal (TMT) joints, although the definition and classifications of the injury have altered over the years $[39,45]$. Indeed, multiple classifications have been presented, yet there is still no consensus on the precise definition of Lisfranc injuries [6, 25, 33]. Nevertheless, Lisfranc injury is recognized nowadays as a wide variety of both bony and ligamentous injuries of the TMT joint region ranging from subtle ligamentous injuries to severely displaced or crush injuries [21, 25, 33, 35, 43, 45].

The incidence of Lisfranc injuries has been presented to be 9.2/100 000 person-years when diagnosed with computed tomography (CT) [34]. Furthermore, it has been estimated 
that even Lisfranc injuries resulting from high-energy trauma mechanisms can be initially overlooked or misdiagnosed in $20 \%-24 \%$ of cases [17, 30]. However, the current literature on the accuracy of the diagnostics is limited. Primary diagnosis is usually based on non-weight-bearing radiographic imaging, though its sensitivity has been estimated to be quite low $(24 \%-50 \%)$ when compared with CT $[17,38]$ Weight-bearing radiographs or magnetic resonance imaging (MRI) are suggested modalities for detecting ligamentous injuries [33, 36-38], yet it may be impossible to acquire weight-bearing images due to the extensively painful foot at the first presentation [33, 36-38, 40, 53]. In their systematic review, Sripanich and colleagues [50] reported that CT scans seem to be currently the most precise imaging modality in detecting bony injuries; whereas, MRI seems to be the most precise in detecting ligamentous injuries. It has also been reported that the sensitivity of the weightbearing radiograph is not higher compared with the non-weight-bearing radiograph and is less sensitive than CT [38]. Nevertheless, many of the previously published studies have still relied on nonweight-bearing or weight-bearing radiographs $[8,9,12,20$, 23, 29, 33, 35, 41, 47].

When evaluating the accuracy of the diagnosis, it is important to evaluate the reliability (interobserver reliability) and the reproducibility (intraobserver reliability) of the diagnostic test [22]. The interobserver reliability is a method to evaluate the correlations between the observers as mathematical measures $[5,19]$. The intraobserver reliability, in turn, is a method to evaluate the test-retest reliability of the diagnostic test [11]. In addition to inter- and intraobserver reliability, it is important to take into account other statistical measures, such as sensitivity, specificity and positive and negative predictive value, when evaluating the accuracy of a diagnostic test $[1,2,10,28]$.

The aim of this study is to assess the inter- and intraobserver reliability and other diagnostic parameters of nonweight-bearing foot radiographs compared with CT in Lisfranc injuries.

\section{Materials and methods}

To assess the accuracy of the diagnostics of Lisfranc injuries, we analysed all foot and ankle CT and CBCT scans acquired due to acute foot trauma at one university hospital and one regional hospital during the period 1.1.2012-31.12.2016. Intra-articular fractures and avulsion fractures around the TMT joint complex were defined as Lisfranc injury. Patients with extra-articular metatarsal injuries were excluded. In addition to the radiologists' report, the CT scans were separately evaluated by two experienced foot surgery experts. In the case of disagreement, the final diagnosis was made together.
In total, the data comprised 456 patients with acute foot injuries. The CT scans revealed 202 patients without any signs of injury, 21 patients with distal metatarsal or toe fractures and 233 patients with a bony injury (joint displacement, intra-articular or avulsion fracture) affecting the Lisfranc joint complex. The patients were divided into displaced and nondisplaced injuries with a threshold of $2 \mathrm{~mm}$ of displacement according to the previous literature [6]. Therefore, injuries with a fracture displacement or TMT joint dislocation of less than $2 \mathrm{~mm}$ were considered to be non-displaced and those with $2 \mathrm{~mm}$ or more were considered to be displaced. Altogether, 174 patients had a nondisplaced Lisfranc injury and 59 patients had a displaced Lisfranc injury. IBM SPSS 24.0 statistical software was used to randomly select 100 patients for the present (reliability) study: 34 patients without a Lisfranc injury (some had distal foot fractures), 33 patients with a non-displaced Lisfranc injury and 33 patients with a displaced Lisfranc injury. The characteristics of the patients are presented in Table 1.

The anonymised primary non-weight-bearing foot radiographs were assessed independently by three senior orthopaedic surgeons (with a minimum of 10 years' experience) and three orthopaedic surgery residents (from 4 to 6 years' experience) twice at intervals of three months. All 100 sets of radiographs were performed in antero-posterior, $30^{\circ}$ oblique and lateral views. The observers were asked to answer the following questions: "Is there an injury at the Lisfranc joint"; (Yes/No), "If you answered yes, describe the findings" and "Are there any other injuries"; (Yes/No).

The sequence of the sets was randomly mixed for the second observation. Picture archiving and communications system (PACS) software was used to display the radiographs.

\section{Statistical analysis}

Fleiss kappa $(\kappa)$ was used to evaluate the interobserver reliability between all six observers in two different moments. Cohen kappa $(\kappa)$ was used to assess the intraobserver reliability between the same observer in two

Table 1 Characteristics of the patients

\begin{tabular}{ll}
\hline & $n=100$ \\
\hline Age, mean (SD) & $40.9(18)$ \\
Males, $n(\%)$ & $55(55 \%)$ \\
Right foot, $n(\%)$ & $58(58 \%)$ \\
Patients with Lisfranc injury & $n=66$ \\
Trauma mechanism, $n(\%)$ & \\
Tumbling or slipping & $25(38)$ \\
Traffic collisions & $11(17)$ \\
Direct injury & $8(6)$ \\
Other & $22(37)$ \\
\hline
\end{tabular}


different moments at an interval of three months. Results were presented according to Landis and Koch criteria: $0.00-0.20$, slight agreement; 0.21-0.40, fair; 0.41-0.60, moderate; 0.61-0.80, substantial; and 0.81-1.00, almost perfect [24]. The clinical characteristics of the patients are presented as means with standard deviations (SD), medians with interquartile ranges (IQR), as counts with percentages, or as ranges. Differences between means of continuous variables were compared with Mann-Whitney test. False-positive rate was calculated as false negatives divided with CT-positive cases, and false-negative rate was calculated by dividing the false-positive cases with CT-negative cases. Microsoft Excel (version 16.15) and $\mathrm{R}$ (version 3.6.0) statistical software were used to conduct statistical analyses.

\section{Results}

When interobserver reliability of non-weight-bearing radiographs in Lisfranc injury was assessed between 6 observers, the $\kappa$ coefficient for interobserver reliability resulted in moderate correlation from $\kappa=0.50$ (95\% CI 0.45-0.55) (first evaluation) to $\kappa=0.58$ (95\% CI $0.52-0.63$ ) (second evaluation). The evaluation was repeated after three months and the $\kappa$ coefficient for intraobserver reliability between the two evaluations of individual observers showed substantial correlation of mean $\kappa=0.71$ (from 0.64 to 0.85 ) (Table 2).

The mean (range) sensitivity of all observers was $76.1 \%$ (60.6-92.4) and specificity was 85.3\% (52.9-100) (Table 2). The sensitivity of the diagnostics in non-displaced injuries was lower than in displaced injuries $(65.4 \%$ vs $87.1 \%$ $p=0.003)$. The number of missed cases was higher among non-displaced injuries than in displaced injuries ( $n=11$ vs 4 $p=0.002$ ). The false-negative rate was $23.9 \%$ and the falsepositive rate was $14.7 \%$. There were no statistically significant differences between senior orthopaedic surgeons and residents in sensitivity $(72.5 \%$ vs. $79.8 \%, p=0.44)$, specificity ( 87.7 vs. $82.8 \%, p=0.92$ ), positive predictive value ( $85.8 \%$ vs. $91.2 \%, p=0.31)$ or negative predictive value (76.5\% vs. $69.4 \%, p=0.31$ ).

Consensus between all evaluators was achieved in 38 (38\%) cases: 26 cases with an injury and 9 cases without an injury were identified correctly by all evaluators during both evaluations. Three cases with a non-displaced Lisfranc injury were missed by all evaluators (Fig. 1a-c). The agreement was compared with the true positive cases detected by CT (Fig. 2). Results demonstrate that a mild consensus was achieved among most of the non-injured patients, without a significant number of false positives. In the case of injured patients, the consensus was not achieved as precisely, and multiple patients were missed by most of the observers.

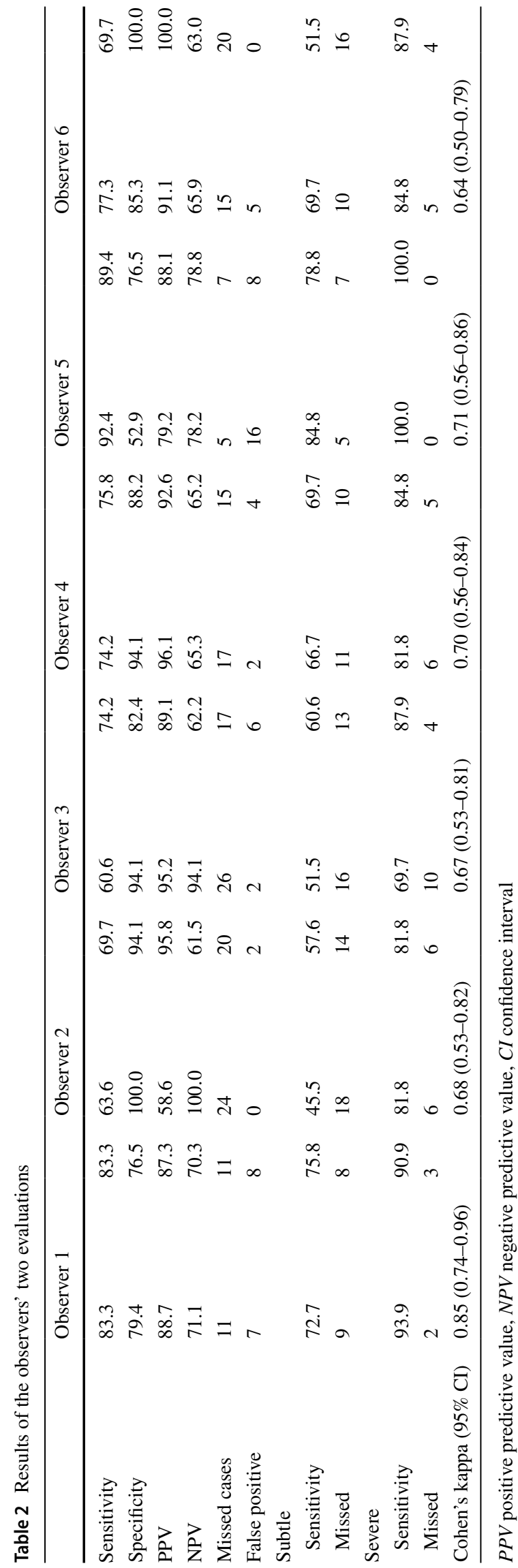


Fig. 1 a-c Radiological findings of the three undisplaced injuries which were missed by all observers. a No specific findings with standard radiographs, yet $\mathrm{CT}$ revealed fractures of the first metatarsal base and medial cuneiform. b No specific findings with standard radiographs, yet avulsion fractures of the second, third and the fourth metatarsal bases were detected in CT. c No specific findings with standard radiographs, yet avulsion fractures of the fourth metatarsal base were detected in CT

\section{Discussion}

The diagnosis of Lisfranc injury based on conventional radiographs had moderate agreement between observers and substantial agreement between the same observer at different time moments. To the best of our knowledge, our study is the first to evaluate the inter- and intraobserver reliability among non-weight-bearing radiographs in the detection of Lisfranc injuries. The main results of our study were that the inter- and intraobserver reliabilities in detecting Lisfranc injuries from non-weight-bearing radiographs depend on the observer, and if the same observer evaluates the same images in different moments, the results will fluctuate. There was some variance in intraobserver reliability among the observers, ranging from substantial agreement to almost perfect. Nondisplaced injuries were significantly more commonly missed than the displaced injuries.

In a previous study by Sherief et al. [48], three radiologists, three orthopaedic surgeons and three physicians evaluated 30 sets of radiographs [48]. The mean sensitivity for Lisfranc injuries was 92\% (95\% CI 89-95\%), and the rate of missed injuries was 19\% [48]. They did not report differences between the radiologists, orthopaedic surgeons or physicians. Haapamäki et al. [16] studied the accuracy of the radiological diagnostics of Lisfranc injuries by comparing the findings of 17 conventional radiographs with CT. They presented a sensitivity of $76 \%$ and a missed injury rate of $24 \%$ for Lisfranc injuries [16]. In addition, Rankine et al. [42] presented a study with 60 non-weight-bearing foot radiographs with 45 CT-positive cases were evaluated by two independent radiologists. They presented a sensitivity of $84.4 \%$, specificity of $53.3 \%$ positive predictive value of $84.4 \%$ and negative predictive value of 53.3\% [42]. In our study with 100 cases, the sensitivity (76\%) was comparable to the numbers presented in earlier studies, where the sensitivity has been between 76 and $92 \%[16,42,48]$. There were no differences between the senior orthopaedic surgeons and residents in our study, a similar finding to the study of Sherief et al. [48].

Instability of the foot arch, seen as widening of the space between the first and second TMT joints, has been suggested to be the main indication to proceed with operative treatment $[3,40,46]$. Previous studies have criticised the accuracy of non-weight-bearing radiographs in the diagnostics of Lisfranc injuries, since they can only reliably detect severe displacement of the Lisfranc joint and instability is difficult
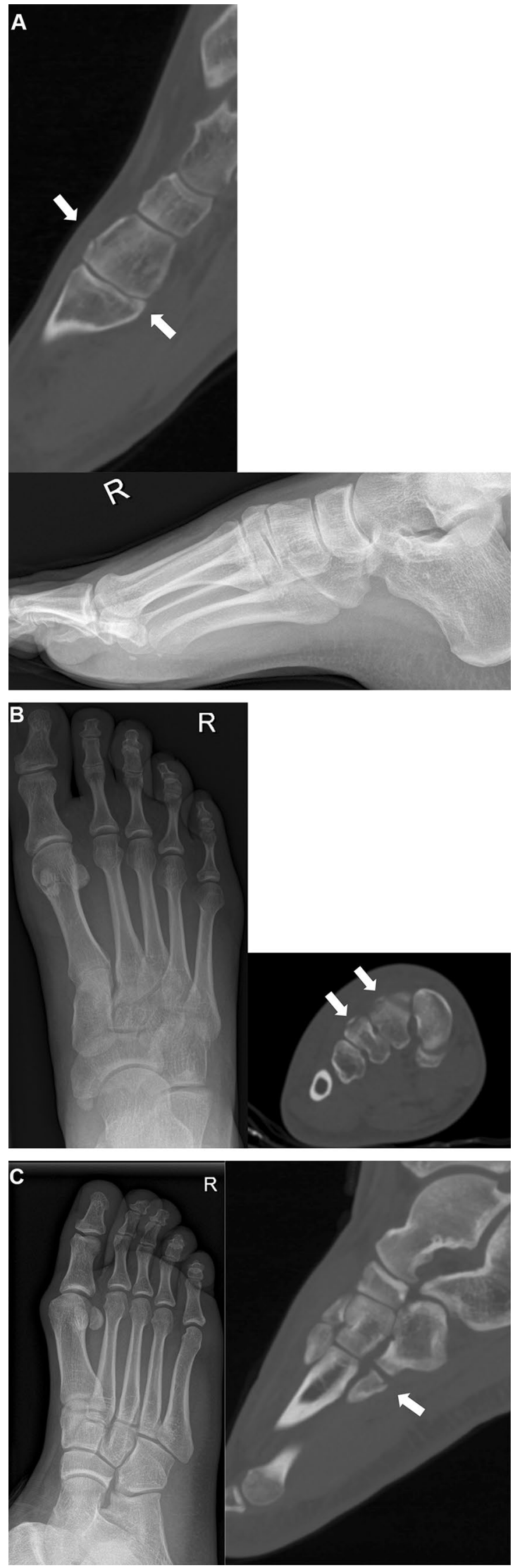


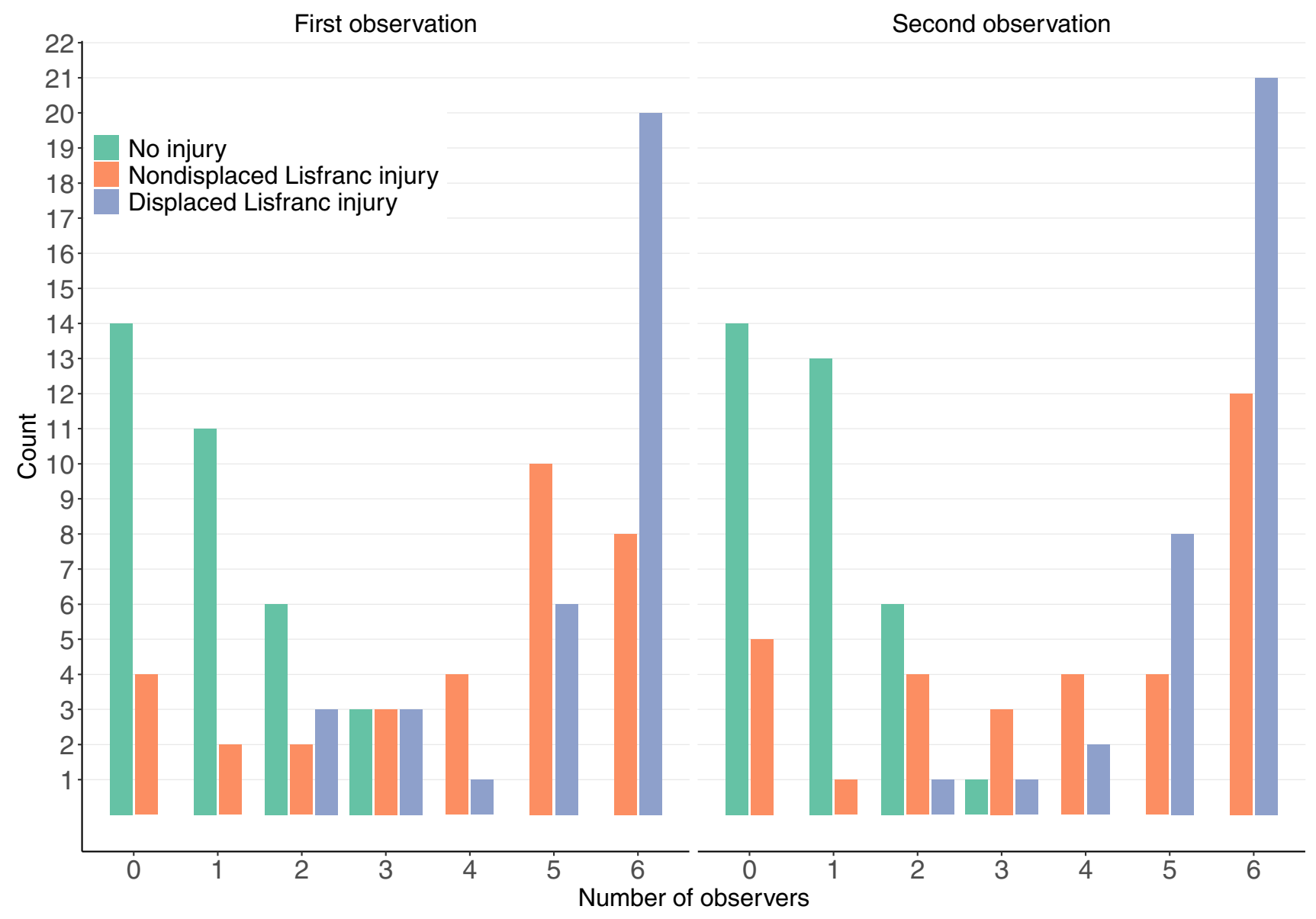

Fig. 2 The distribution of the agreement between the observers. Green bars indicate that the non-injured patients were detected with relatively high consensus. Blue (displaced) and orange (nondisplaced) bars represent the agreement between the patients with Lisfranc injury

to assess $[13,15,32,51]$. To correct this flaw, it has been suggested that weight-bearing radiographs are used $[3,7,9$, 15]. However, the problem with weight-bearing radiographs is that the severity of pain usually prevents the patients from reliably bearing weight, and therefore it is impossible to obtain reproducible images [50].

The study by Goiney et al. [14] was the first to describe the benefits of using $\mathrm{CT}$ over non-weight-bearing radiography. Since then, the advantages of CT have attracted more interest $[26,38]$. The biggest benefit of $\mathrm{CT}$ is that small bony displacements, avulsion fragments and fractures are detectable; whereas, they would be missed in non-weight-bearing radiography [26]. To the best of our knowledge, the only study comparing these different imaging modalities in the same sample of Lisfranc injuries was performed by Preidler et al. [38]. They compared the differences between conventional radiography, weight-bearing radiography, CT and MRI with a sample of 49 patients. Their conclusion was that weight-bearing radiographs or MRI do not provide any additional benefit when compared with conventional radiography, and that $\mathrm{CT}$ is the most sensitive imaging modality for detecting Lisfranc injuries.
The previous literature provides at least 15 different classification systems for Lisfranc injuries [18, 25, 30, 33, 45, 49]. Injury classifications should be developed as tools to help doctors in decision-making and in choosing the optimal treatment for each patient [4]. Accurately working classifications should also provide estimates of the outcomes after the chosen treatment [4]. In addition, the classifications should have a high inter- and intraobserver reliability to ensure reliability and responsiveness [4]. The inter- and intraobserver reliabilities have been evaluated for the radiograph-based Hardcastle [18] and Myerson [30] classifications for dislocated Lisfranc injuries $[27,52]$. Moreover, the inter- and intraobserver reliabilities for these classifications have varied from moderate to excellent [27, 52]. Since most of the previous classifications are based on nonweight-bearing radiographs, we feel it is essential to evaluate the reliability and responsiveness of this imaging modality.

As the use of CT as a diagnostic tool has gained more popularity, the most recently published classifications for Lisfranc injuries have been based on CT imaging [25, 45]. The most recent CT-based classification, the Column Involvement Severity System by Schepers and Rammelt [45], divides 
Lisfranc injuries according to the columns of the midfoot. The classification represents the affected columns: medial, central and lateral, with the severity of the injury, classified as 0 -no joint involved, 1 - pure ligamentous with avulsions, 2 - simple fracture and 3-comminuted fracture. They suggest that instability is evaluated either by weight-bearing radiographs or stress radiographs under anaesthesia one week after the injury. However, as previously stated, neither of these modalities has been shown to be reliable in detecting the instability $[31,38]$. In addition, this classification does not help to choose between nonoperative or operative treatment or to predict the outcome after the chosen treatment.

The strength of our study was the large data sample that included a broad range of Lisfranc injuries. Since the term 'Lisfranc injury' is indicative of a wide variety of different injuries in terms of severity, displacement and number of affected joints, it is essential to evaluate the diagnostics with an appropriate study sample [18, 30, 33, 43]. The limitation of our study was that the radiographs were only evaluated by orthopaedic surgeons and orthopaedic surgery residents who are familiar with Lisfranc injuries. However, most of the initial diagnostics occurs in primary healthcare, and patients are then referred to specialized medical care units. Hence, the initial evaluation is often performed by general physicians and it can be assumed that the precision of the diagnostics may be even weaker than the results presented in this work. In addition, the lack of using MRI, weight-bearing CT or weight-bearing radiographs can be considered as a limitation, since some patients with purely ligamentous injuries could be missed.

Since our results show that a significant number of patients would be missed by conventional radiographs, we feel that it is essential to confirm the diagnosis with CT imaging if the clinical suspicion of the injury is high (plantar ecchymosis, pain in active and passive movements or swelling) [9, 44]. Furthermore, there is a need for an accurate injury classification for Lisfranc injuries, based on $\mathrm{CT}$, that would help the clinician with the decision-making and would predict the outcomes after the chosen treatment. Although the classification by Schepers and Rammelt [45] has introduced a novel approach to these injuries, it still requires some further evaluation before it can be used as a tool for choosing the correct treatment for patients.

To conclude, the radiologic diagnosis of a Lisfranc injury based on conventional radiographs has moderate agreement between observers and substantial agreement between the same observer in different time moments. The sensitivity and reliability for detecting Lisfranc injuries with conventional radiographs indicated relatively moderate accuracy. In other words, a substantial number (24\%) of injuries are missed if only non-weight-bearing radiographs are used.

Acknowledgements We wish to thank the Vappu Uuspää Foundation, the Finnish Research Foundation for Orthopaedics and Traumatology and the Competitive State Research Financing of the Expert Responsibility area of Tampere University Hospital (grant number 9R006 and R9031) for the financial support of this study.

Funding This study was financially supported by Vappu Uuspää Foundation, Finnish Research Foundation for Orthopaedics and Traumatology and the Competitive State Research Financing of the Expert Responsibility area of Tampere University Hospital, Grant number 9R006 and R9031.

\section{Compliance with ethical standards}

Conflict of interest The authors declare that they have no conflict of interest.

Ethical approval This article does not contain any studies with human participants or animals performed by any of the authors.

Open Access This article is licensed under a Creative Commons Attribution 4.0 International License, which permits use, sharing, adaptation, distribution and reproduction in any medium or format, as long as you give appropriate credit to the original author(s) and the source, provide a link to the Creative Commons licence, and indicate if changes were made. The images or other third party material in this article are included in the article's Creative Commons licence, unless indicated otherwise in a credit line to the material. If material is not included in the article's Creative Commons licence and your intended use is not permitted by statutory regulation or exceeds the permitted use, you will need to obtain permission directly from the copyright holder. To view a copy of this licence, visit http://creativecommons.org/licenses/by/4.0/.

\section{References}

1. Altman DG, Bland JM (1994) Diagnostic tests. 1: sensitivity and specificity. BMJ 308(6943):1552

2. Altman DG, Bland JM (1994) Statistics notes: diagnostic tests 2: predictive values. BMJ 309(6947): 102

3. Arntz CT, Veith RG, Hansen ST Jr (1988) Fractures and fracture-dislocations of the tarsometatarsal joint. J Bone Joint Surg 70(2):173-181

4. Burstein AH (1993) Do they work and are they useful? JBJS 75(12):1743-1744

5. Caro T, Roper R, Young M, Dank G (1979) Inter-observer reliability. Behaviour 69(3):303-315

6. Chiodo CP, Myerson MS (2001) Developments and advances in the diagnosis and treatment of injuries to the tarsometatarsal joint. Orthop Clin N Am 32(1):11-20

7. Coss LHS, Usnr MC, Manos LRE et al (1998) Abduction stress and AP weightbearing radiography of purely ligamentous Injury in the tarsometatarsal joint. Foot Ankle Int 19(8):537-541

8. Crates JM, Barber FA, Sanders EJ (2015) Subtle Lisfranc subluxation: results of operative and nonoperative treatment. J Foot Ankle Surg 54(3):350-355

9. Curtis MJ, Myerson M, Szura B (1993) Tarsometatarsal joint injuries in the athlete. Am J Sports Med 21(4):497-502

10. Deeks JJ (2001) Systematic reviews of evaluations of diagnostic and screening tests. BMJ 323(7305):157-162

11. Eliasziw M, Young SL, Woodbury MG, Fryday-Field K (1994) Statistical methodology for the concurrent assessment of interrater and intrarater reliability: using goniometric measurements as an example. Phys Ther 74(8):777-788 
12. Faciszewski T, Burks RT, Manaster BJ (1990) Subtle injuries of the Lisfranc joint. J Bone Joint Surg 72(10):1519-1522

13. Foster SC, Foster RR (1976) Lisfranc's tarsometatarsal fracturedislocation. Radiology 120(1):79-83

14. Goiney RC, Connell DG, Nichols DM (1985) CT evaluation of tarsometatarsal fracture-dislocation injuries. AJR Am J Roentgenol 144(5):985-990

15. Goossens M, Stoop ND (1983) Lisfranc's Fracture-Dislocations: Etiology, Radiology, and Results of Treatment. Clin Orthop Relat Res 176:154-162

16. Haapamaki V, Kiuru M, Koskinen S (2004) Lisfranc fracturedislocation in patients with multiple trauma: diagnosis with multidetector computed tomography. Foot Ankle Int 25(9):614-619

17. Haapamaki VV, Kiuru MJ, Koskinen SK (2004) Ankle and foot injuries: analysis of MDCT findings. AJR Am J Roentgenol 183(3):615-622

18. Hardcastle PH, Reschauer R, Kutscha-Lissberg E, Schoffmann W (1982) Injuries to the tarsometatarsal joint. Incidence, classification and treatment. J Bone Joint Surg 64(3):349-356

19. Hartmann DP (1977) Considerations in the choice of interobserver reliability estimates. J Appl Behav Anal 10(1):103-116

20. Henning JA, Jones CB, Sietsema DL, Bohay DR, Anderson JG (2009) Open reduction internal fixation versus primary arthrodesis for lisfranc injuries: a prospective randomized study. Foot Ankle Int 30(10):913-922

21. Kosters C, Bockholt S, Muller C et al (2014) Comparing the outcomes between Chopart, Lisfranc and multiple metatarsal shaft fractures. Arch Orthop Trauma Surg 134(10):1397-1404

22. Krippendorff K (2008) Reliability. The international encyclopedia of communication. Wiley, Hoboken

23. Kuo RS, Tejwani NC, Digiovanni CW et al (2000) Outcome after open reduction and internal fixation of Lisfranc joint injuries. J Bone Joint Surg 82:1609-1618

24. Landis JR, Koch GG (1977) The measurement of observer agreement for categorical data. Biometrics 33:159-174

25. Lau SC, Guest C, Hall M, Tacey M, Joseph S, Oppy A (2017) Do columns or sagittal displacement matter in the assessment and management of Lisfranc fracture dislocation? An alternate approach to classification of the Lisfranc injury. Injury 30:30

26. Lu J, Ebraheim NA, Skie M, Porshinsky B, Yeasting RA (1997) Radiographic and computed tomographic evaluation of Lisfranc dislocation: a cadaver study. Foot Ankle Int 18(6):351-355

27. Mahmoud S, Hamad F, Riaz M, Ahmed G, Al Ateeq M, Ibrahim T (2015) Reliability of the Lisfranc injury radiological classification (Myerson-modified Hardcastle classification system). Int Orthop 39(11):2215-2218

28. Mallett S, Halligan S, Thompson M, Collins GS, Altman DG (2012) Interpreting diagnostic accuracy studies for patient care. BMJ 345:e3999

29. Mulier T, Reynders P, Dereymaeker G, Broos P (2002) Severe Lisfrancs injuries: primary arthrodesis or ORIF? Foot Ankle Int 23(10):902-905

30. Myerson MS, Fisher RT, Burgess AR, Kenzora JE (1986) Fracture dislocations of the tarsometatarsal joints: end results correlated with pathology and treatment. Foot Ankle 6(5):225-242

31. Naguib S, Meyr AJ (2018) Reliability, surgeon preferences, and eye-tracking assessment of the stress examination of the tarsometatarsal (Lisfranc) joint complex. J Foot Ankle Surg 58:93-96

32. Norfray JF, Geline RA, Steinberg RI, Galinski AW, Gilula L (1981) Subtleties of Lisfranc fracture-dislocations. Am J Roentgenol 137(6):1151-1156

33. Nunley JA, Vertullo CJ (2002) Classification, investigation, and management of midfoot sprains. Am J Sports Med 30(6):871-878

34. Ponkilainen VT, Laine H-J, Mäenpää HM, Mattila VM, Haapasalo HH (2018) Incidence and characteristics of midfoot injuries. Foot Ankle Int 40(1):105-112
35. Porter DA, Barnes AF, Rund A, Walrod MT (2018) Injury pattern in ligamentous Lisfranc injuries in competitive athletes. Foot Ankle Int 40(2):185-194

36. Potter HG, Deland JT, Gusmer PB, Carson E, Warren RF (1998) Magnetic resonance imaging of the Lisfranc ligament of the foot. Foot Ankle Int 19(7):438-446

37. Preidler KW, Brossmann J, Daenen B, Goodwin D, Schweitzer M, Resnick D (1996) MR imaging of the tarsometatarsal joint: analysis of injuries in 11 patients. Am J Roentgenol 167(5):1217-1222

38. Preidler KW, Peicha G, Lajtai G et al (1999) Conventional radiography, CT, and MR imaging in patients with hyperflexion injuries of the foot: diagnostic accuracy in the detection of bony and ligamentous changes. Am J Roentgenol 173(6):1673-1677

39. Quenu EKG (1909) Etude sur les luxations du metatarse (Luxations metatarso-tarsiennes). Du diastasis entre le 1er et le 2e metatarsien. Rev Chir 39:1-72

40. Raikin SM, Elias I, Dheer S, Besser MP, Morrison WB, Zoga AC (2009) Prediction of midfoot instability in the subtle Lisfranc injury: comparison of magnetic resonance imaging with intraoperative findings. JBJS 91(4):892-899

41. Rajapakse B, Edwards A, Hong T (2006) A single surgeon's experience of treatment of Lisfranc joint injuries. Injury 37(9):914-921

42. Rankine JJ, Nicholas CM, Wells G, Barron DA (2012) The diagnostic accuracy of radiographs in Lisfranc injury and the potential value of a craniocaudal projection. Am J Roentgenol 198(4):W365-W369

43. Renninger CH, Cochran G, Tompane T, Bellamy J, Kuhn K (2017) Injury characteristics of low-energy Lisfranc injuries compared with high-energy injuries. Foot Ankle Int 38(9):964-969

44. Ross G, Cronin R, Hauzenblas J, Juliano P (1996) Plantar ecchymosis sign: a clinical aid to diagnosis of occult Lisfranc tarsometatarsal injuries. J Orthop Trauma 10(2):119-122

45. Schepers T, Rammelt $S$ (2018) Classifying the Lisfranc injury: Literature overview and a new classification. Fuß Sprunggelenk $16: 151-159$

46. Seo DK, Lee HS, Lee KW, Lee SK, Kim SB (2017) Nonweightbearing radiographs in patients with a subtle Lisfranc injury. Foot Ankle Int 38(10):1120-1125

47. Shapiro MS, Wascher DC, Finerman GA (1994) Rupture of Lisfranc's ligament in athletes. Am J Sports Med 22(5):687-691

48. Sherief TI, Mucci B, Greiss M (2007) Lisfranc injury: how frequently does it get missed? And how can we improve? Injury 38(7):856-860

49. Sivakumar BS, An VV, Oitment C, Myerson M (2018) Subtle Lisfranc injuries: a topical review and modification of the classification system. Orthopedics 41(2):e168-e175

50. Sripanich Y, Weinberg MW, Krähenbühl N et al (2019) Imaging in Lisfranc injury: a systematic literature review. Skelet Radiol 49:1-23

51. Stein RE (1983) Radiological aspects of the tarsometatarsal joints. Foot Ankle 3(5):286-289

52. Talarico RH, Hamilton GA, Ford LA, Rush SM (2006) Fracture dislocations of the tarsometatarsal joints: analysis of interrater reliability in using the modified Hardcastle classification system. J Foot Ankle Surg 45(5):300-303

53. Vuori JP, Aro HT (1993) Lisfranc joint injuries: trauma mechanisms and associated injuries. J Trauma-Injury Inf Crit Care 35(1):40-45

Publisher's Note Springer Nature remains neutral with regard to jurisdictional claims in published maps and institutional affiliations. 